\title{
Skew Category Algebras
}

\author{
V. V. Bavula
}

Received: 29 November 2018 / Revised: 10 April 2019 / Accepted: 12 April 2019 / Published online: 13 November 2019

(C) The Author(s) 2019

\begin{abstract}
We study a new (large) class of algebras (that was introduced in Bavula in Math Comput Sci 11(34):253-268, 2017) — the skew category algebras. Any such an algebra $\mathcal{C}(\sigma)$ is constructed from a category $\mathcal{C}$ and a functor $\sigma$ from the category $\mathcal{C}$ to the category of algebras. Criteria are given for the algebra $\mathcal{C}(\sigma)$ to be simple or left Noetherian or right Noetherian or semiprime or have 1.
\end{abstract}

Keywords A skew category algebra $\cdot$ A simple algebra $\cdot$ A left Noetherian algebra $\cdot$ A semiprime algebra

Mathematics Subject Classification $16 \mathrm{P} 40 \cdot 16 \mathrm{~S} 35 \cdot 16 \mathrm{~S} 34 \cdot 16 \mathrm{P} 60 \cdot 16 \mathrm{~N} 60$

\section{Skew Category Algebras, Examples and Constructions}

In this paper, $K$ is a commutative ring with 1 , algebra means a $K$-algebra. In general, it is not assumed that a $K$-algebra has an identity element. Module means a left module. Missed definitions can be found in [1].

Let $\mathcal{C}$ be a category, $\operatorname{Ob}(\mathcal{C})$ be the set of its objects and $\operatorname{Mor}(\mathcal{C})$ be the set of its morphisms. For each objects $i, j \in \mathrm{Ob}(\mathcal{C}), \mathcal{C}(i, j)$ is the set of morphisms $f: i \rightarrow j$, the objects $i=t(f)$ and $j=h(f)$ are called the tail and head of the morphism $f$, respectively. For each object $i \in \mathrm{Ob}(\mathcal{C}), e_{i}$ is the identity morphism $i \rightarrow i$.

Definition 1.1 ([2]) Let $\mathcal{C}$ be a category and $\sigma$ be a functor from the category $\mathcal{C}$ to the category of unital $K$-algebras over a commutative ring $K$ (eg, $K=\mathbb{Z}$ or $K$ is a field). So, for each object $i \in \operatorname{Ob}(\mathcal{C}), D_{i}:=\sigma(i)$ is a $K$-algebra and for each morphism

$f: i \mapsto j, \quad \sigma_{f}: D_{i} \rightarrow D_{j}$

is a $K$-algebra homomorphism, and $\sigma_{f g}=\sigma_{f} \sigma_{g}$ for all morphisms $f$ and $g$ such that $t(f)=h(g)$. The direct sum of left $K$-modules

$\mathcal{C}(\sigma)=\bigoplus_{f \in \operatorname{Mor}(\mathcal{C})} D_{h(f)} f$

V. V. Bavula $(\bowtie)$

School of Mathematics and Statistics, University of Sheffield, Hicks Building, Sheffield S3 7RH, UK

e-mail: v.bavula@sheffield.ac.uk 
where $D_{h(f)} f$ is a free left $D_{h(f)}$-module of rank 1, is a $K$-algebra with multiplication given by the rule: For all $f, g \in \operatorname{Mor}(\mathcal{C}), a \in D_{h(f)}$ and $b \in D_{h(g)}$,

$a f \cdot b g= \begin{cases}a \sigma_{f}(b) f g & \text { if } t(f)=h(g), \\ 0 & \text { otherwise. }\end{cases}$

It is a trivial exercise to verify that the multiplication is associative. The $K$-algebra $\mathcal{C}(\sigma)$ is called a skew category $K$-algebra. If $K=\mathbb{Z}$, the $\mathbb{Z}$-algebra $\mathcal{C}(\sigma)$ is called a skew category ring.

Definition 1.2 If the direct sum (1) admits an associative product which is given by the rule: For all $f, g \in \operatorname{Mor}(\mathcal{C})$, $a \in D_{h(f)}$ and $b \in D_{h(g)}$,

$a f \cdot b g= \begin{cases}a \sigma_{f}(b) c(f, g) f g & \text { if } t(f)=h(g), \\ 0 & \text { otherwise, }\end{cases}$

where

$c(f, g) \in \begin{cases}D_{h(f)} & \text { if } t(f)=h(g), \\ \{0\} & \text { otherwise, }\end{cases}$

then it is called the twisted skew category $K$-algebra and is denoted by $\mathcal{C}(\sigma, c)$.

The categorical nature of the above classes of rings especially the categorical/explicit nature of their multiplications makes these classes important as far as various computational aspects are concerned.

Let $1_{i}$ be the identity of the algebra $D_{i}$. Then $1_{i} e_{i} \in D_{i} e_{i} \subseteq \mathcal{C}(\sigma)$ where $i \in \operatorname{Ob}(\mathcal{C})$. Abusing the notation, we write $e_{i}$ for $1_{i} e_{i}$. Then $e_{i} \in \mathcal{C}(\sigma)$.

The $\mathcal{C}$-grading on $\mathcal{C}(\sigma)$. By the very definition, the algebra $\mathcal{C}(\sigma)$ is a $\mathcal{C}$-graded algebra, that is

$D_{h(f)} f \cdot D_{h(g)} g \subseteq D_{h(f g)} f g$ for all $f, g \in \operatorname{Mor}(\mathcal{C})$.

The algebra $\mathcal{C}(\sigma)$ is a direct sum

$\mathcal{C}(\sigma)=\bigoplus_{i, j \in \mathrm{Ob}(\mathcal{C})} \mathcal{C}(\sigma)_{i j}$ where $\mathcal{C}(\sigma)_{i j}=\bigoplus_{f \in \mathcal{C}(j, i)} D_{i} f$

and for all $i, j, k, l \in \mathrm{Ob}(\mathcal{C})$,

$\mathcal{C}(\sigma)_{i j} \mathcal{C}(\sigma)_{k l} \subseteq \delta_{j k} \mathcal{C}(\sigma)_{i l}$

where $\delta_{j k}$ is the Kronecker delta. In particular, for each $i \in \mathrm{Ob}(\mathcal{C}), \mathcal{C}(\sigma)_{i i}$ is a $K$-algebra without 1 , in general. For each $i, j \in \operatorname{Ob}(\mathcal{C}), \mathcal{C}(\sigma)_{i j}$ is a $\left(\mathcal{C}(\sigma)_{i i}, \mathcal{C}(\sigma)_{j j}\right)$-bimodule.

The next two examples show that even for two simplest categories that contain a single object, a single loop or a single invertible loop, the above construction gives apart from a skew polynomial ring or a skew Laurent polynomial ring, new classes of rings.

Example 1 Let $\mathcal{C}$ be a category that contains a single object, say 1 , and $\operatorname{Mor}(\mathcal{C})=\left\{x^{i} \mid i \in \mathbb{N}\right\}$ where $e:=x^{0}$ is the identity morphism. So, $\mathcal{C}(\sigma)=D e \oplus D x \oplus \cdots \oplus D x^{i} \oplus \cdots$ where $D=\sigma(1)$ and $e d=\sigma_{e}(d) e$ and $x^{i} d=\sigma_{x}^{i}(d) x^{i}$ for all $i \geq 1$ where $\sigma_{e}$ and $\sigma_{x}$ are ring endomorphisms of $D$ such that $\sigma_{e} \sigma_{x}=\sigma_{x} \sigma_{e}=\sigma_{x}$ and $\sigma_{e}^{2}=\sigma_{e}$.

- If $\sigma_{e}=\operatorname{id}_{D}$ then $\mathcal{C}(\sigma)=D\left[x ; \sigma_{x}\right]$ is a skew polynomial ring.

- If $\sigma_{e} \neq \operatorname{id}_{D}$ then $\mathcal{C}(\sigma)$ is not a skew polynomial ring since $e d=\sigma_{e}(d) e$ and, in general, $\sigma_{e}(d) e \neq d e$ for all $d \in D\left(\right.$ since $\left.\sigma_{e} \neq \mathrm{id}_{D}\right)$. For example, let $D=D_{1} \times D_{2} \times D_{3}$ and $\sigma_{e}$ and $\sigma_{x}$ are the projections onto $D_{1} \times D_{2}$ and $D_{1}$, respectively. Then $e D_{3}=0$.

Example 2 Let $\mathcal{C}$ be a category that contains a single object, say 1 , and $\operatorname{Mor}(\mathcal{C})=\left\{x^{i} \mid i \in \mathbb{Z}\right\}$ where $e:=x^{0}$ is the identity morphism $\left(x x^{-1}=x^{-1} x=e\right)$. The functor $\sigma$ is determined by the algebra $D=\sigma(1)$ and its algebra endomorphisms $\sigma_{e}, \sigma_{x}$ and $\sigma_{x^{-1}}$ such that

$\sigma_{e}^{2}=\sigma_{e}, \quad \sigma_{e} \sigma_{x^{ \pm 1}}=\sigma_{x^{ \pm 1}} \sigma_{e}=\sigma_{x^{ \pm 1}}$ and $\sigma_{x} \sigma_{x^{-1}}=\sigma_{x^{-1}} \sigma_{x}=\sigma_{e}$.

Then $\mathcal{C}(\sigma)=\oplus_{i \in \mathbb{Z}} D x^{i}$. 
- If $\sigma_{e}=\operatorname{id}_{D}$ then $\sigma_{x^{-1}}=\sigma_{x}^{-1}$ and $\mathcal{C}(\sigma)=D\left[x^{ \pm 1} ; \sigma_{x}\right]$ is a skew Laurent polynomial ring.

- If $\sigma_{e} \neq \operatorname{id}_{D}$ then $\mathcal{C}(\sigma)$ is not a skew Laurent polynomial ring. For example, let $D=D_{1} \times D_{2}$ be a direct product of algebras and $\sigma_{e}=\sigma_{x}=\sigma_{x^{-1}}$ be the projection onto $D_{1}$. Then $e D_{2}=0$ and $x D_{2}=x^{-1} D_{2}=0$.

Example 3 Let $\mathcal{C}$ be a category that contains a single object, say 1 , and the monoid $\mathcal{C}(1,1)$ is generated by elements $x$ and $y$ subject to the defining relation $y x=e$. The functor $\sigma$ is determined by the algebra $D=\sigma(1)$ and its three algebra endomorphisms $\sigma_{x}, \sigma_{y}$ and $\sigma_{e}$ such that

$\sigma_{y} \sigma_{x}=\sigma_{e}$.

The skew category algebra $\mathcal{C}(\sigma)$ is called the skew semi-Laurent polynomial ring [2]. It is a new class of rings. Suppose, for simplicity, that $\sigma_{e}=\mathrm{id}_{D}$. Then the ring $\mathcal{C}(\sigma)$ is generated by a ring $D$ and elements $x$ and $y$ subject to the defining relations:

$y x=1, \quad x d=\sigma_{x}(d) x$ and $y d=\sigma_{y}(d) y$ for all $d \in D$.

We denote this ring by $D\left[x, y ; \sigma_{x}, \sigma_{y}\right]$. In particular, $D\left[x, y ; \tau, \tau^{-1}\right]$ where $\tau$ is an automorphism of $D$.

Example 4 Let $n \geq 1$ be a natural number and $\mathcal{M}_{n}$ be the matrix units category:

$\operatorname{Ob}\left(\mathcal{M}_{n}\right)=\{1, \ldots, n\}, \mathcal{M}_{n}(i, j)=\left\{E_{j i}\right\}$ and $E_{i j} E_{j k}=E_{i k}$ for all $i, j, k$.

Let $D$ be a ring and $f_{1}, \ldots, f_{n}$ be its automorphisms. Define $\sigma$ by the rule $\sigma(i)=D$ and $\sigma\left(E_{i j}\right)=f_{i} f_{j}^{-1}$. The skew category algebra

$\mathcal{M}_{n}(\sigma)=\oplus_{i, j=1}^{n} D E_{i j}$

is called the skew matrix ring where the multiplication is given by the rule

$d E_{i j} \cdot d^{\prime} E_{k l}=\delta_{j k} d f_{i} f_{j}^{-1}\left(d^{\prime}\right) E_{j l}$ for all $d, d^{\prime} \in D$.

The skew graph rings and the skew tree rings.

Definition 1.3 ([2]) Let $\Gamma=\left(\Gamma_{0}, \Gamma_{1}\right)$ be a non-oriented graph without cycles where $\Gamma_{0}$ is the set of vertices and $\Gamma_{1}$ is the set of edges. If, in addition, $\Gamma$ is connected then it is called a tree. So, any non-oriented graph without cycles is a disjoint union of its connected components which are trees. Let $\Gamma$ be the category groupoid associated with $\Gamma$ : $\mathrm{Ob}(\boldsymbol{\Gamma})=\Gamma_{0}$, for each $i \in \mathrm{Ob}(\Gamma), \boldsymbol{\Gamma}(i, i)=\left\{e_{i i}\right\}$, for distinct $i, j \in \mathrm{Ob}(\boldsymbol{\Gamma})$ such that $(i, j) \in \Gamma_{1}, \boldsymbol{\Gamma}(i, j)=\left\{e_{j i}\right\}$ and $\boldsymbol{\Gamma}(j, i)=\left\{e_{i j}\right\}, e_{i j} e_{j i}=e_{i i}$ and $e_{j i} e_{i j}=e_{j j}$. Let $\sigma$ be a functor from $\boldsymbol{\Gamma}$ to the category of rings. Then $\boldsymbol{\Gamma}(\sigma)$ is called the skew graph ring. If $\Gamma$ is a tree then $\Gamma(\sigma)$ is called the skew tree ring. We say that the functor $\sigma$ is of isomorphism type if $\sigma\left(e_{i j}\right): \sigma(i) \rightarrow \sigma(j)$ is a unital ring isomorphism for all $(i, j) \in \Gamma_{1}$.

Theorem 1.4 Let $\Gamma$ be a finite tree, $n=\left|\Gamma_{0}\right|$ and the functor $\sigma$ be of isomorphism type. Suppose that for some $i \in \Gamma_{0}$ the ring $D_{i}=\sigma(i)$ is a semiprime, left (resp., right) Goldie ring and $Q_{l}\left(D_{i}\right)$ (resp., $Q_{r}\left(D_{i}\right)$ ) is its left (resp., right) quotient ring. Then $\Gamma(\sigma)$ is a semiprime, left (resp., right) Goldie ring and $Q_{l}(\Gamma(\sigma)) \simeq M_{n}\left(Q_{l}\left(D_{i}\right)\right)$ (resp., $Q_{r}(\Gamma(\sigma)) \simeq M_{n}\left(Q_{r}\left(D_{i}\right)\right)$ ) where $M_{n}(R)$ is a matrix ring over a ring $R$. In particular, the left (resp., right) uniform dimension of $\boldsymbol{\Gamma}(\sigma)$ is $n d_{l}$ (resp., $n d_{r}$ ) where $d_{l}$ (resp., $d_{r}$ ) is a left (resp., right) uniform dimension of $D_{i}$.

Proof (Sketch). Let $\mathcal{C}_{D_{j}}$ be the set of regular elements of the ring $D_{j}=\sigma(j)$. All the rings $D_{j}$ are isomorphic. The set of regular elements $S=\oplus_{j=1}^{n} \mathcal{C}_{D_{j}} e_{j j}$ is a left Ore set of $\Gamma(\sigma)$ such that $S^{-1} \Gamma(\sigma)$ is a semisimple Artinian ring. Furthermore, $S^{-1} \Gamma(\sigma) \simeq M_{n}\left(Q_{l}\left(D_{i}\right)\right)$. Hence, $Q_{l}(\Gamma(\sigma)) \simeq M_{n}\left(Q_{l}\left(D_{i}\right)\right)$, and so $\Gamma(\sigma)$ is a semiprime, left Goldie ring. The rest is obvious.

As a result we have the following corollary.

Corollary 1.5 Let $\Gamma$ be a finite non-orientable graph, i.e., $\Gamma=\coprod_{s=1}^{v} \Gamma^{(s)}$ is a disjoint union of finite trees $\Gamma^{(s)}$. Then 
1. The skew graph ring $\Gamma(\sigma)$ is a direct product $\prod_{s=1}^{v} \Gamma^{(s)}\left(\sigma_{s}\right)$ of skew tree rings where $\sigma_{s}$ is the restriction of the functor $\sigma$ to $\Gamma^{(s)}\left(\sigma_{s}\right)$.

2. If the trees $\Gamma^{(s)}(s=1, \ldots, v)$ satisfy the conditions of Theorem 1.4 then $Q_{l}(\Gamma(\sigma)) \simeq \prod_{s=1}^{v} Q_{l}\left(\Gamma^{(s)}\left(\sigma_{s}\right)\right)$ (resp., $Q_{r}(\Gamma(\sigma)) \simeq \prod_{s=1}^{v} Q_{r}\left(\Gamma^{(s)}\left(\sigma_{s}\right)\right)$ ) is a direct product of semiprime, left (resp., right) Goldie rings, and so it is a semiprime, left (resp., right) Goldie ring.

\section{Properties of Skew Category Algebras}

In this section, criteria are given for a skew category algebra $\mathcal{C}(\sigma)$ to be left/right Noetherian or semiprime or simple.

The ideal $\mathfrak{a}$ and the algebra $\overline{\mathcal{C}(\sigma)}$.

Lemma 2.1 Let $D$ be a ring and $\sigma^{\prime}$ be its ring endomorphism such that $\sigma^{\prime 2}=\sigma^{\prime}$. Then $D=\sigma^{\prime}(D) \oplus \operatorname{ker}\left(\sigma^{\prime}\right)$ and the restriction homomorphism $\left.\sigma^{\prime}\right|_{\sigma^{\prime}(D)}: \sigma^{\prime}(D) \rightarrow \sigma^{\prime}(D), d \mapsto d$ is the identity automorphism.

Proof Straightforward.

By (5), the formal sum

$e=\sum_{i \in \mathrm{Ob}(\mathcal{C})} e_{i}$

determines two well-defined maps:

$e \cdot: \mathcal{C}(\sigma) \rightarrow \mathcal{C}(\sigma), \quad a \mapsto e a$ and $\cdot e: \mathcal{C}(\sigma) \rightarrow \mathcal{C}(\sigma), a \mapsto a e$.

Clearly, the map $\cdot e$ is the identity map id on $\mathcal{C}(\sigma)$ but the kernel $\mathfrak{a}$ of the map $e$. is equal to

$\mathfrak{a}(\mathcal{C}(\sigma)):=\mathfrak{a}:=\bigoplus_{f \in \operatorname{Mor}(\mathcal{C})} \mathfrak{a}_{h(f)} f$

where $\mathfrak{a}_{i}:=\operatorname{ker}\left(\sigma_{e_{i}}\right)$ and $\sigma_{i}:=\sigma_{e_{i}}: D_{i} \rightarrow D_{i}$ is a $K$-algebra endomorphism, and $(e \cdot)^{2}=e \cdot$. Since $\sigma_{i}^{2}=\sigma_{i}$,

$D_{i}=\sigma_{i}(D) \oplus \mathfrak{a}_{i}$ for all $i \in \mathrm{Ob}(\mathcal{C})$,

by Lemma 2.1 .

$\mathcal{C}(\sigma)=\overline{\mathcal{C}(\sigma)} \oplus \mathfrak{a}$ where $\overline{\mathcal{C}(\sigma)}:=\bigoplus_{f \in \operatorname{Mor}(\mathcal{C})} \sigma_{h(f)}\left(D_{h(f)}\right) f$

is a $K$-subalgebra of $\mathcal{C}(\sigma)$ such that the maps $\left.(e \cdot)\right|_{\overline{\mathcal{C}}(\sigma)}: \overline{\mathcal{C}(\sigma)} \rightarrow \overline{\mathcal{C}(\sigma)}, c \mapsto c$ and $\left.(\cdot e)\right|_{\overline{\mathcal{C}}(\sigma)}: \overline{\mathcal{C}(\sigma)} \rightarrow \overline{\mathcal{C}(\sigma)}$, $c \mapsto c$ are the identity map on $\overline{\mathcal{C}(\sigma)}$.

Lemma 2.2 The set $\mathfrak{a}$ is an ideal of the algebra $\mathcal{C}(\sigma)$ such that $\mathcal{C}(\sigma) \mathfrak{a}=0, \mathfrak{a} \mathcal{C}(\sigma)=\mathfrak{a}$ and $\mathfrak{a}^{2}=0$.

Proof $\mathcal{C}(\sigma) \mathfrak{a}=\mathcal{C}(\sigma) \cdot e \cdot \mathfrak{a}=0$, the rest is obvious.

The next theorem shows that the algebra $\overline{\mathcal{C}(\sigma)}$ is also a skew category algebra.

Theorem 2.3 1. The subalgebra $\overline{\mathcal{C}(\sigma)}$ of $\mathcal{C}(\sigma)$ is also a skew category algebra $\overline{\mathcal{C}(\sigma)}=\mathcal{C}(\bar{\sigma})$ where for each $i \in \operatorname{Ob}(\mathcal{C}), \bar{\sigma}(i):=\sigma_{i}\left(D_{i}\right)$ and for each $f \in \mathcal{C}(i, j), \bar{\sigma}_{f}:=\left.\sigma_{f}\right|_{\sigma_{i}\left(D_{i}\right)}: \sigma_{i}\left(D_{i}\right) \rightarrow \sigma_{i}\left(D_{i}\right), d \mapsto \sigma_{f}(d)$.

2. For all $i \in \mathrm{Ob}(\mathcal{C}), \bar{\sigma}_{i}=\mathrm{id}_{\bar{\sigma}(i)}$.

3. $\mathfrak{a}(\mathcal{C}(\bar{\sigma}))=0$.

4. The maps $e \cdot$ and $\cdot e$ are the identity maps on $\mathcal{C}(\bar{\sigma})$.

Proof 1. Statement 1 follows from (8) and the fact that $\sigma_{j} \sigma_{f}=\sigma_{f}=\sigma_{f} \sigma_{i}$ for all elements $f \in \mathcal{C}(i, j)$. $2-4$. Statement 2 is obvious. Then statements 3 and 4 follow from statement 2. 
The ideal $\mathfrak{a}$ is a $\mathcal{C}$-graded ideal of the algebra $\mathcal{C}(\sigma)$. Furthermore,

$$
\mathfrak{a}=\bigoplus_{i, j \in \mathrm{Ob}(\mathcal{C})} \mathfrak{a}_{i j}
$$

where $\mathfrak{a}_{i j}=\bigoplus_{f \in \mathcal{C}(j, i)} \mathfrak{a}_{i} f \subseteq \mathcal{C}(\sigma)_{i j}, 0=\mathfrak{a}_{i j} \mathfrak{a}_{k l} \subseteq \delta_{j k} \mathfrak{a}_{i l}$ for all $i, j, k, l \in \mathrm{Ob}(\mathcal{C})$. Since $\overline{\mathcal{C}(\sigma)}=\mathcal{C}(\bar{\sigma})$ (Theorem 2.3.(1)), the factor algebra

$\overline{\mathcal{C}(\sigma)}=\mathcal{C}(\sigma) / \mathfrak{a}=\bigoplus_{f \in \operatorname{Mor}(\mathcal{C})} \bar{D}_{h(f)} f \subseteq \mathcal{C}(\sigma)$

is a $\mathcal{C}$-graded algebra where $\bar{D}_{i}=D_{i} / \mathfrak{a}_{i}=\operatorname{im}\left(\sigma_{i}\right)$. Furthermore,

$\overline{\mathcal{C}(\sigma)}=\bigoplus_{\mathbf{i}, j \in \operatorname{Ob}(\mathcal{C})}{\overline{\mathcal{C}}(\sigma)_{i j}}_{i j}$ where $\overline{\mathcal{C}(\sigma)_{i j}}=\mathcal{C}(\sigma)_{i j} / \mathfrak{a}_{i j}$

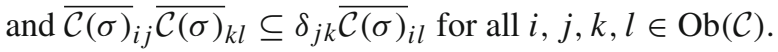

Theorem 2.4 (Criterion for $\mathcal{C}(\sigma)$ to be a left Noetherian algebra) The algebra $\mathcal{C}(\sigma)$ is a left Noetherian algebra iff the following conditions hold

1. the set $\mathrm{Ob}(\mathcal{C})$ is a finite set,

2. the ideal $\mathfrak{a}$ is a finitely generated abelian group,

3. for every object $i \in \mathrm{Ob}(\mathcal{C})$, the $K$-algebra ${\overline{\mathcal{C}}(\sigma)_{i i}}_{i}$ is a left Noetherian algebra, and

4. for all objects $i, j \in \mathrm{Ob}(\mathcal{C})$ such that $i \neq j$, the left ${\overline{\mathcal{C}}(\sigma)_{i i}}_{- \text {module }}{\overline{\mathcal{C}}(\sigma)_{i j}}_{i}$ is finitely generated.

Proof The algebra $\mathcal{C}(\sigma)=\bigoplus_{j \in \mathrm{Ob}(\mathcal{C})} \mathcal{C}(\sigma)_{* j}$ is a direct sum of nonzero left ideals where

$\mathcal{C}(\sigma)_{* j}:=\bigoplus_{i \in \operatorname{Ob}(\mathcal{C})} \mathcal{C}(\sigma)_{i j}$

So, the algebra $\mathcal{C}(\sigma)$ is a left Noetherian algebra iff the set $\mathrm{Ob}(\mathcal{C})$ is a finite set and all the left ideals $\mathcal{C}(\sigma)_{* j}$ are Noetherian left $\mathcal{C}(\sigma)$-modules iff $|\mathrm{Ob}(\mathcal{C})|<\infty$, the left $\mathcal{C}(\sigma)$-module $\mathfrak{a}$ is Noetherian and all the left $\overline{\mathcal{C}(\sigma)}$-modules

$\overline{\mathcal{C}(\sigma)}_{* j}=\bigoplus_{i \in \operatorname{Ob}(\mathcal{C})}{\overline{\mathcal{C}}(\sigma)_{i j}}$

are Noetherian (since $\mathcal{C}(\sigma)=\overline{\mathcal{C}(\sigma)} \oplus \mathfrak{a}$ is a direct sum of left $\mathcal{C}(\sigma)$-modules) iff conditions 1 and 2 hold (since $\mathcal{C}(\sigma) \mathfrak{a}=0$, Lemma 2.4) and the left ${\overline{\mathcal{C}}(\sigma)_{i i}}_{\text {-module }}{\overline{\mathcal{C}}(\sigma)_{i j}}_{i j}$ is Noetherian for all $i, j \in \mathrm{Ob}(\mathcal{C})$ (since each left $\overline{\mathcal{C}(\sigma)}$-submodule $M$ of ${\overline{\mathcal{C}}(\sigma)_{* j}}_{\text {is a direct sum }}$

$M=e M=\bigoplus_{i \in \operatorname{Ob}(\mathcal{C})} e_{i} M$

where $e_{i} M$ is a left $\overline{\mathcal{C}(\sigma)}_{i i}$-submodule of $\overline{\mathcal{C}(\sigma)}_{i j}$ and the functor from the category of all ${\overline{\mathcal{C}}(\sigma)_{i i}}_{\text {-submodules of }}$ ${\overline{\mathcal{C}}(\sigma)_{i j}}_{i}$ to the category of all $\overline{\mathcal{C}(\sigma)}$-submodules of ${\overline{\mathcal{C}}(\sigma)_{* j}}_{\text {, }}$

$N \rightarrow \overline{\mathcal{C}(\sigma)} N=\bigoplus_{k \in \mathrm{Ob}(\mathcal{C})} \overline{\mathcal{C}}(\sigma)_{k i} N$

is faithful since $e_{i} \overline{\mathcal{C}(\sigma)} N={\overline{\mathcal{C}}(\sigma)_{i i}} N=N$ ) iff statements $1-4$ hold.

Proposition 2.5 (Criterion for $\mathcal{C}(\sigma)$ to be a right Noetherian algebra) The algebra $\mathcal{C}(\sigma)$ is a right Noetherian algebra iff the following conditions hold

1. the set $\mathrm{Ob}(\mathcal{C})$ is a finite set,

2. for every object $i \in \mathrm{Ob}(\mathcal{C})$, the $K$-algebra $\mathcal{C}(\sigma)_{i i}$ is a right Noetherian algebra, and 
3. for all objects $i, j \in \mathrm{Ob}(\mathcal{C})$ such that $i \neq j$, the right $\mathcal{C}(\sigma)_{j j}$-module $\mathcal{C}(\sigma)_{i j}$ is finitely generated.

Proof The algebra $\mathcal{C}(\sigma)=\bigoplus_{i \in \mathrm{Ob}(\mathcal{C})} \mathcal{C}(\sigma)_{i *}$ is a direct sum of nonzero right ideals where

$\mathcal{C}(\sigma)_{i *}=\bigoplus_{j \in \mathrm{Ob}(\mathcal{C})} \mathcal{C}(\sigma)_{i j}$

So, the algebra $\mathcal{C}(\sigma)$ is a right Noetherian algebra iff the set $\mathrm{Ob}(\mathcal{C})$ is a finite set and all right ideals $\mathcal{C}(\sigma)_{i *}$ are Noetherian right $\mathcal{C}(\sigma)$-modules iff $|\mathrm{Ob}(\mathcal{C})|<\infty$ and the right $\mathcal{C}(\sigma)_{j j}$-module $\mathcal{C}(\sigma)_{i j}$ is Noetherian for all $i, j \in \mathrm{Ob}(\mathcal{C})$ iff $|\mathrm{Ob}(\mathcal{C})|<\infty$, the rings $\mathcal{C}(\sigma)_{i i}$ are right Noetherian and the right $\mathcal{C}(\sigma)_{j j}$-modules $\mathcal{C}(\sigma)_{i j}$ are finitely generated for all $i \neq j$.

Example 5 Let $\mathcal{C}: 1 \stackrel{f}{\rightarrow} 2$ and the functor $\sigma$ is as follows: $\sigma(1)=\mathbb{Q}, \sigma(2)=\mathbb{R}, \sigma_{e_{1}}=\mathrm{id}_{\mathbb{Q}}, \sigma_{e_{2}}=\operatorname{id}_{\mathbb{R}}$ and $\sigma_{f}: \mathbb{Q} \rightarrow \mathbb{R}, q \mapsto q$. Then the algebra $\mathcal{C}(\sigma)$ is isomorphic to the lower triangular matrix algebra $\left(\begin{array}{ll}\mathbb{Q} & 0 \\ \mathbb{R} & \mathbb{R}\end{array}\right)$. By Theorem 2.4, the algebra $\mathcal{C}(\sigma)$ is left Noetherian but not right Noetherian, by Proposition 2.5 (since $\mathbb{R}_{\mathbb{Q}}$ is not a finitely generated right $\mathbb{Q}$-module).

Example 6 Let $\mathcal{C}: 1 \stackrel{f}{\rightarrow} 2$ and the functor $\sigma$ is as follows: $\sigma(1)=K[t]$ is a polynomial algebra in the variable $t$ over $K, \sigma(2)=K, \sigma_{e_{1}}: K[t] \rightarrow K[t], t \mapsto 0 ; \sigma_{e_{2}}=\operatorname{id}_{K}: K \rightarrow K$ and $\sigma_{f}: K[t] \rightarrow K, t \mapsto 0$. Then $\mathfrak{a}=t K[t] e_{1}$ is not a finitely generated $\mathbb{Z}$-module. So, the algebra $\mathcal{C}(\sigma)$ is not a left Noetherian algebra, by Theorem 2.4. Since the algebra $\mathcal{C}(\sigma)_{11}=K[t] e_{1}$ is not a right Noetherian algebra, the ring $\mathcal{C}(\sigma)$ is not a right Noetherian ring, by Proposition 2.5.

Lemma 2.6 (Existence of 1 in $\mathcal{C}(\sigma)$ ) The algebra $\mathcal{C}(\sigma)$ has 1 iff the set $\mathrm{Ob}(\mathcal{C})$ is a finite set and $\sigma_{e_{i}}=\operatorname{id}_{D_{i}}$ for all $i \in \mathrm{Ob}(\mathcal{C})$. In this case, $e=\sum_{i \in \mathrm{Ob}(\mathcal{C})} e_{i}$ is the identity of the algebra $\mathcal{C}(\sigma)$.

Proof $(\Rightarrow)$ Suppose that 1 is an identity of $\mathcal{C}(\sigma)$. Then necessarily the set $\mathrm{Ob}(\mathcal{C})$ is a finite set, otherwise $1 a=0$ for some nonzero element $a$ of $\mathcal{C}(\sigma)$. The $1=\sum_{i, j} 1_{i j}$ where $1_{i j} \in \mathcal{C}(\sigma)_{i j}$. The equalities $1 e_{j}=e_{j}=e_{j} 1$ for all $j \in \mathrm{Ob}(\mathcal{C})$ imply that $1=\sum_{i \in \mathrm{Ob}(\mathcal{C})} e_{i}=e$. Then, necessarily $\sigma_{e_{i}}=\operatorname{id}_{D_{i}}$ for all $i \in \mathrm{Ob}(\mathcal{C})$.

$(\Leftarrow)$ Clearly, $e$ is the identity of the algebra $\mathcal{C}(\sigma)$.

Lemma 2.7 Suppose that $n=|\mathrm{Ob}(\mathcal{C})|<\infty$. If I is an ideal of $\mathcal{C}(\sigma)$ such that $e_{i} I e_{i}=0$ for all $i \in \mathrm{Ob}(\mathcal{C})$ then $I^{n+1}=0$.

Proof By $(8), \mathcal{C}(\sigma)=\overline{\mathcal{C}(\sigma)} \oplus \mathfrak{a}$. Hence, $I \subseteq \bar{I} \oplus \mathfrak{a}$ where $\bar{I}=(I+\mathfrak{a}) / \mathfrak{a}=\sum_{i, j \in \mathrm{Ob}(\mathcal{C})} e_{i} I e_{j} \subseteq \overline{\mathcal{C}(\sigma)}$. Notice that $\bar{I}^{n}=0$ since $e_{i} I e_{i}=0$ for all $i \in \mathrm{Ob}(\mathcal{C})$. Now,

$I^{n+1} \subseteq(\bar{I}+\mathfrak{a})^{n+1} \subseteq \bar{I}^{n+1}+\mathfrak{a} \bar{I}^{n}=0$

since $\mathfrak{a}^{2}=0$ and $\mathcal{C}(\sigma) \mathfrak{a}=0$ (Lemma 2.2).

Recall that a ring is a semiprime ring if the zero ideal is the only nilpotent ideal.

Theorem 2.8 (Criterion for $\mathcal{C}(\sigma)$ to be a semiprime algebra) Suppose that $n:=|\mathrm{Ob}(\mathcal{C})|<\infty$. Then the following statements are equivalent.

1. The algebra $\mathcal{C}(\sigma)$ is a semiprime algebra.

2. The algebras $\mathcal{C}(\sigma)_{i i}$ are semiprime where $i \in \mathrm{Ob}(\mathcal{C})$ and, for all distinct $i, j \in \mathrm{Ob}(\mathcal{C}), a_{i j} \mathcal{C}(\sigma)_{j i} \neq 0$ and $\mathcal{C}(\sigma)_{j i} a_{i j} \neq 0$ for all nonzero elements $a_{i j} \in \mathcal{C}(\sigma)_{i j}$.

3. The algebras $\mathcal{C}(\sigma)_{i i}$ are semiprime where $i \in \mathrm{Ob}(\mathcal{C})$ and each ideal $I$ of $\mathcal{C}(\sigma)$ such that $e_{i} I e_{i}=0$ for all $i \in \mathrm{Ob}(\mathcal{C})$ is equal to zero. 
Proof Since $|\mathrm{Ob}(\mathcal{C})|<\infty$, the direct product of algebras $\mathcal{D}:=\prod_{i \in \mathrm{Ob}(\mathcal{C})} \mathcal{C}(\sigma)_{i i}$ is a semiprime algebra iff all the algebras $\mathcal{C}(\sigma)_{i i}$ are semiprime.

$(1 \Rightarrow 2)$ If $\mathfrak{b}$ is a nonzero nilpotent ideal of the ring $\mathcal{D}$ and $(\mathfrak{b})=\mathcal{C}(\sigma) \mathfrak{b C}(\sigma)$ is the ideal of $\mathcal{C}(\sigma)$ generated by $\mathfrak{b}$ then

$(\mathfrak{b})^{k} \subseteq\left(\mathfrak{b}^{\left\lfloor\frac{k}{n^{2}}\right\rfloor}\right)$ for all $k \geq 1$

where for a real number $r,\lfloor r\rfloor:=\max \{z \in \mathbb{Z} \mid z \leq r\}$, and so the ideal (b) of the algebra $\mathcal{D}$ is a nilpotent ideal. Therefore, the ring $\mathcal{C}(\sigma)_{i i}$ must be semiprime for all $i \in \mathrm{Ob}(\mathcal{C})$.

Suppose that there exists a nonzero element $a_{i j} \in \mathcal{C}(\sigma)_{i j}$ for some distinct objects $i$ and $j$ such that either $a_{i j} \mathcal{C}(\sigma)_{j i}=0$ or $\mathcal{C}(\sigma)_{j i} a_{i j}=0$. Then $\left(a_{i j}\right)^{2}=\left(a_{i j} \mathcal{C}(\sigma)_{j i} a_{i j}\right)=0$, a contradiction.

(2 $\Rightarrow 1$ ) Since all rings $\mathcal{C}(\sigma)_{i i}$ are semiprime, the ideal $\mathfrak{a}$ is equal to zero, by Lemma 2.2. Therefore, if $J$ is a nilpotent ideal of $\mathcal{C}(\sigma)$ then necessarily $J=\bigoplus_{i, j \in \operatorname{Ob}(\mathcal{C})} J_{i j}$ where $J_{i j}=e_{i} J e_{j}$. Furthermore, all $J_{i i}=0$ since the rings $\mathcal{C}(\sigma)_{i i}$ are semiprime (and $J_{i i}^{m} \subseteq J^{m}$ for all $m \geq 1$ ). Suppose that $J \neq 0$. We seek a contradiction. Then $J_{i j} \neq 0$ for some $i \neq j$. Then, by the assumption, either $\mathcal{C}(\sigma)_{j i} J_{i j}$ is a nonzero nilpotent ideal of the algebra $\mathcal{C}(\sigma)_{j j}$ or $J_{i j} \mathcal{C}(\sigma)_{j i}$ is a nonzero nilpotent ideal of the algebra $\mathcal{C}(\sigma)_{i i}$, a contradiction.

$(1 \Rightarrow 3)$ The algebras $\mathcal{C}(\sigma)_{i i}$ are semiprime for all $i \in \mathrm{Ob}(\mathcal{C})$, by the implication $(1 \Rightarrow 2)$. By Lemma 2.7, each ideal $I$ of $\mathcal{C}(\sigma)$ such that $e_{i} I e_{i}=0$ for all $i \in \mathrm{Ob}(\mathcal{C})$ is a nilpotent ideal, so it must be zero (since $\mathcal{C}(\sigma)$ is a semiprime ring).

$(3 \Rightarrow 1)$ If $I$ is a nilpotent ideal of $\mathcal{C}(\sigma)$ then for each $i \in \operatorname{Ob}(\mathcal{C}), I_{i i}$ is a nilpotent ideals of the semiprime ring $\mathcal{C}(\sigma)_{i i}$, and so $I_{i i}=0$. Then, we must have $I=0$, by the second assumption of statement 3 .

Theorem 2.9 (Simplicity criterion for $\mathcal{C}(\sigma)$ ) The algebra $\mathcal{C}(\sigma)$ is a simple algebra iff the following conditions hold

1. $\mathfrak{a}=0$,

2. for every $i \in \mathrm{Ob}(\mathcal{C})$, the ring $\mathcal{C}(\sigma)_{i i}$ is simple,

3. for all distinct $i, j \in \mathrm{Ob}(\mathcal{C}), \mathcal{C}(\sigma)_{i j}$ is a simple $\left(\mathcal{C}(\sigma)_{i i}, \mathcal{C}(\sigma)_{j j}\right)$-bimodule (in particular, $\left.\mathcal{C}(\sigma)_{i j} \neq 0\right)$, and

4. $\mathcal{C}(\sigma)_{i j} \mathcal{C}(\sigma)_{j k} \neq 0$ for all $i, j, k \in \mathrm{Ob}(\mathcal{C})$.

Proof $(\Rightarrow)$ Let $\mathcal{C}_{i j}=\mathcal{C}(\sigma)_{i j}$.

(i) $\mathfrak{a}=0$, by Lemma 2.2 .

(ii) For every $i \in \operatorname{Ob}(\mathcal{C}), \mathcal{C}_{i i}$ is a simple ring: Suppose that $\mathfrak{b}$ is a proper ideal of the $\operatorname{ring} \mathcal{C}_{i i}$ then (b) is a proper ideal of $\mathcal{C}(\sigma)$ since $(\mathfrak{b}) \cap \mathcal{C}_{i i}=\mathfrak{b}$, a contradiction.

(iii) For all distinct objects $i, j \in \mathrm{Ob}(\mathcal{C}), \mathcal{C}_{i j} \neq 0$ : Suppose that $\mathcal{C}_{i j}=0$ for some distinct objects $i$ and $j$. Then the ideal $\left(\mathcal{C}_{i i}\right)$ of $\mathcal{C}(\sigma)$ is a proper ideal since $\left(\mathcal{C}_{i i}\right) \cap \mathcal{C}_{j j}=\mathcal{C}_{j i} \mathcal{C}_{i i} \mathcal{C}_{i j}=0$, a contradiction.

(iv) For all distinct objects $i, j \in \operatorname{Ob}(\mathcal{C}), \mathcal{C}_{i j}$ is a simple $\left(\mathcal{C}_{i i}, \mathcal{C}_{j j}\right)$-bimodule: Suppose that $\mathfrak{b}$ is a proper $\left(\mathcal{C}_{i i}, \mathcal{C}_{j j}\right)$ sub-bimodule of $\mathcal{C}_{i j}$ then $(\mathfrak{b})$ is a proper ideal of the algebra $\mathcal{C}(\sigma)$ since $(\mathfrak{b}) \cap \mathcal{C}_{i j}=\mathfrak{b}$, a contradiction.

(v) $\mathcal{C}_{i j} \mathcal{C}_{j k} \neq 0$ for all objects $i, j, k \in \mathrm{Ob}(\mathcal{C})$ : The statement (v) holds in the following cases $i=j=k$ (by (ii)), $i=j$ or $j=k$ (by (iii)). Suppose that $i=k$ and $\mathcal{C}_{i j} \mathcal{C}_{j i}=0$, we seek a contradiction. Then the ideal $\left(\mathcal{C}_{i j}\right)$ of $\mathcal{C}(\sigma)$ is a proper ideal since $\left(\mathcal{C}_{i j}\right) \cap \mathcal{C}_{i i}=\mathcal{C}_{i j} \mathcal{C}_{j i}=0$, a contradiction. Suppose that $\mathcal{C}_{i j} \mathcal{C}_{j k}=0$ for some distinct $i, j$ and $k$. Then the ideal $\left(\mathcal{C}_{i j}\right)$ of $\mathcal{C}(\sigma)$ is a proper ideal since $\left(\mathcal{C}_{i j}\right) \cap \mathcal{C}_{k k}=\mathcal{C}_{k i} \mathcal{C}_{i j} \mathcal{C}_{j k}=0$, a contradiction.

$(\Leftarrow)$ Suppose that conditions 1-4 hold. By conditions 1-3, condition 4 can be replaced by condition $4^{\prime}: \mathcal{C}_{i j} \mathcal{C}_{j k}=\mathcal{C}_{i k}$ for all $i, j, k \in \operatorname{Ob}(\mathcal{C})$. Let $J$ be a nonzero ideal of $\mathcal{C}(\sigma)$. We have to show that $J=\mathcal{C}(\sigma)$. By condition $1, e_{i} J e_{j} \neq 0$ for some $i$ and $j$. By conditions 2 and $3, J_{i j}=J \cap \mathcal{C}_{i j}=\mathcal{C}_{i j}$. By condition $4^{\prime}, \mathcal{C}_{s t}=\mathcal{C}_{s i} \mathcal{C}_{i j} \mathcal{C}_{j t} \subseteq J$ for all $s, t$. This means that $J=\mathcal{C}(\sigma)$, as required.

Open Access This article is distributed under the terms of the Creative Commons Attribution 4.0 International License (http:// creativecommons.org/licenses/by/4.0/), which permits unrestricted use, distribution, and reproduction in any medium, provided you give appropriate credit to the original author(s) and the source, provide a link to the Creative Commons license, and indicate if changes were made. 


\section{References}

1. McConnell, J.C., Robson, J.C.: Noncommutative Noetherian rings. With the Cooperation of L. W. Small. Revised edition. Graduate Studies in Mathematics, vol. 30. American Mathematical Society, Providence (2001)

2. Bavula, V.V.: Quiver generalized Weyl algebras, skew category algebras and diskew polynomial rings. Math. Comput. Sci. 11(3-4), 253-268 (2017)

Publisher's Note Springer Nature remains neutral with regard to jurisdictional claims in published maps and institutional affiliations. 